\title{
Falling Forward: The Fermi paradox, supermassive black holes, and the likely flight of extraterrestrial intelligence toward extreme time dilation.
}

\author{
Chris Reiss \\ christopher.j.reiss@gmail.com
}

February 26, 2023

\begin{abstract}
It is argued that an advanced extraterrestrial intelligence has a compelling incentive to permanently occupy a reference frame where time is greatly slowed by relativistic effects. We then show that a near orbit around a supermassive black hole provides a viable - and perhaps only - possibility for such a migration. The expected character of radio emissions from such a civilization is discussed to suggest a method of potential detection.
\end{abstract}

\section{Introduction}

We suggest a resolution to the Fermi paradox, and propose a method which may be uniquely capable of detecting radio emissions from an Extra Terrestrial Intelligence (ETI). Suppose there exists an advanced ETI that, like us, is intrinsically curious. It seeks to explore the Cosmos, to discover life and to communicate with other ETIs. The form such a civilization might take has long been a topic of speculation.

Kardashev postulated that civilizations would tend to advance by consuming 1 exponentially increasing energy. Their appetite would accelerate until it darkens whole galaxies.

In this, Kardashev presupposes that energy is a critical constraint to an interstellar life form. This is a reasonable assumption, but one which evokes a lingering suspicion that we - as early 21st century humans struggling to transition from chemical to nuclear energy - are guilty of mistaking our current problems for the more fundamental and enduring problems likely to confront a much more advanced civilization.

Before we speculate as to the form an ETI civilization might take, we pause to consider : What would their biggest problem necessarily be? We seek the most universal limitations that would obstruct a civilization's ambition to explore and communicate. That is, limitations inherent to the laws of the space-time they share with us.

\footnotetext{
${ }^{1}$ Sinces mass-energy is conserved, by 'consume' we mean 'redirect'.
} 
Apart from its curiosity, we further assume that this sentience experiences time in a manner similar to humans. For us humans, a 1-second wait is barely noticable, a 1-minute wait is a mere pause, a 1-hour wait is a chance to do something else, a year-long wait strains our intuitive grasp, a 100 year wait is essentially 'never'. We assume the ETI sentience also experiences time this way (within an order or two of magnitude) and shares with us, if not our mortality, then at least some degree of our impatience. ${ }^{2}$

From this vantage, the cosmic speed limit of $\boldsymbol{c}$ appears the primary obstacle to a curious ETI. If an ETI on the other side of our Milky Way were to send a signal to Earth we would not receive it for 100,000 years. They would have to wait 200,000 years for the reply.

Special Relativity does, of course, allow for an explorer from that ETI to travel to Earth in a week, day, or any arbitrarily small duration as measured by that explorer. But 100,000 years will still have elapsed on Earth during the trip, and 200,000 will have elapsed on their home world upon their return. It has been suggested that a wormhole or other topological anomaly may be discovered or created in order to circumvent this universal speed limit. We propose another approach - rather surprising in its simplicity - which compensates for the $\boldsymbol{c}$-limit but does not rely on exotic (and undiscovered) features of space-time.

\section{Definition of a red-frame}

While the phenomenon of time dilation is familiar enough, we typically think of it in terms of a traveller whose 'home time' is a planet or structure where clocks tick at roughly the same rate as on Earth. In this article we propose a drastic change in perspective where the 'home time' itself is untethered from its natural origin and deliberately slowed by orders of magnitude. The entire civilization has made a permanent transition and now experiences external events differently.

Such time-frames exist within our current understanding of General Relativity. They arise from motion at very high speeds (close to $\boldsymbol{c}$ ), or from very intense gravity induced by a nearby massive object. For the purposes of this discussion,we shall refer to a reference-frame which is much slower than our own as a 'red-frame'. Frames with similar time-rates as ours we shall call 'blueframes'.

We set practical considerations aside momentarily for the sake of a thought experiment in which we explore the prospects and perspective of a hypothetical red-frame civilization whose time is slowed by a factor of 100x.

\section{Advantages of a red-frame : New prospects for explo- ration, communication and production}

We assumed at the outset a race of beings who, like us, seek to explore and engage with distant worlds. As seen from their new red-frame home, distant objects take on a new character. External clocks are seen to run 100x faster and - of particular interest - external distances contract by 100x

\footnotetext{
${ }^{2}$ We aren't referring to the sentient's life-span necessarily; we can imagine a sentience like ourselves granted immortality but still possessed of natural impatience; living forever doesn't make asn eon pass by faster. One can argue in favor of this assumption by observing it holds across species of life on earth, that exo-life is chemically likely to rise as carbon-based creatures similar to us )
} 
according to a local ruler. 3 The Cosmos has drawn in all around them; their sky is ablaze with the blue-shifted light of stars 100 times closer.

It becomes evident that a red-frame civilization is vastly more capable of exploration. A radio signal can envelope the entire Milky Way in a mere 500 years; a response could be detected within 1,000. It is the same for physical exploration at near light-speed. ${ }^{4}$

Other advantages also emerge. The relative acceleration of time in any exterior blue-frame can be harnessed to the red-frame's benefit. Any productive process performed in a blue-frame becomes 100x faster. This accelerates many forms of work an ETI might wish to undertake: computation, harvesting energy, assembly of mega-structures, terra-forming of planets, and so on.

Such a blue-frame productive 'colony' can accelerate minds as well as machines. Some portion of this ETI race could 'drop down' to a nearby blue-frame in order to conduct scientific research and other intellectual labor. Their red-frame home would be the happy recipient of a century's progress delivered annually.

\section{Orbit of a red-frame}

We consider how our hypothetical red-frame might be actualized by an advanced civilization, by means of an artificial mega-structure (or natural body which is intentionally accelerated.)

General Relativity provides for an arbitrary degree of time dilation at very high speeds and in intense gravity. Intuition suggests that we maximize both by placing the red-frame in near orbit around a supermassive black hole (SMBH). In the Discussion and Conclusions section we justify this guess, eliminating other possibilities in favor of such an orbit as the best - and possibly only case to consider.

Here we simply turn our attention to the supermassive black hole at the center of the Milky Way, Sagittarius-A* (Sgr A*)

We make the simplifying assumption that Sgr A* is a Schwarzschild black hole (i.e., has no spin nor electric charge.) We take the mass to be $4.1 \mathrm{M}$ Solar Masses [Schoeder et al. (2003)] yielding a Schwarzschild Radius $R_{s}=12 \times 10^{9} \mathrm{~m}$

The Innermost Stable Circular Orbit (ISCO) is located at $R_{s c o}=36 \times 10^{9} \mathrm{~m}$.

The photon sphere radius is $R_{p h}=18 \times 10^{9} \mathrm{~m}$

A satellite in the ISCO of a Schwarzschild black hole of any mass has a time dilation factor of $\gamma=\sqrt{2}$ [see Eq. (1) below]. Since we seek a more extreme dilation of $\gamma=100$, we must consider orbits that are much closer than the ISCO and are thus unstable.

These orbits would require constant minute corrections to their speed in order to hold their orbit. (The energy expenditure is arbitrarily small if orbital drift is detected quickly enough.) The

\footnotetext{
${ }^{3}$ To clarify : They would observe a beam of light originating from their red-frame and reflected off a planet 500 light years away (by a blue-frame's ruler) to return in just 10 years local (proper) time. General Relativity cautions that this is not an illusory effect of time dilation; observations from the world-line of the red-frame have no less claim to reality or primacy than observations made from Earth.

${ }^{4}$ The advantage is not to the explorer travelling near $c$, but rather the society that sent them. The home world won't have to wait eons for their explorer to return.
} 
observation of a satellite in steady circular orbit in the unstable region between $R_{p h}$ and $R_{s c o}$ is suggestive of a technosignature due to the inability of a natural satellite to hold such an orbit.

For a satellite in circular Keplerian orbit at radius $r$ around a Schwartzschild black hole, the time dilation factor $\gamma$ is found by multiplying the gravitational red-shift $1 / \sqrt{g_{t t}}$ by the Lorentz boost $\frac{g_{t t}}{1-3 M / r}$ where $g_{t t}=1-2 M / r$ to give

$$
\gamma=\sqrt{\frac{r}{r-3 M}}
$$

[see, e.g., Opatrný et al. (2017)]

Solving for $r$ gives $r=3 M \frac{1}{1-1 / \gamma^{2}}$ which we prefer to write as :

$$
r=R_{p h}+\frac{R_{p h}}{\gamma^{2}-1}
$$

A plot of Eq.(2) illustrates that orbits of significant time-dilation lie very near the photon sphere :

Figure 1

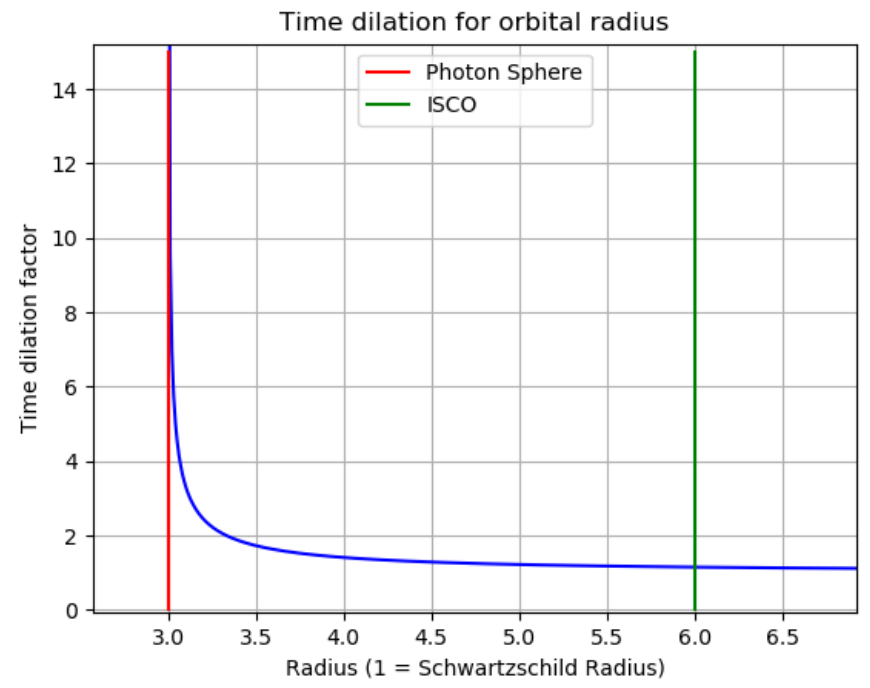

We define a 'red-satellite' as an orbiter with time dilation $\gamma>10$. 
By way of example, we find the circular orbit around Sag A* where time dilation would slow clocks by a factor of $\gamma=100$. Referring to $R_{p h}=18 \mathrm{M} \mathrm{Km}$ and Eq.(2) we find the corresponding orbit at $r=R_{p h}+1800 \mathrm{Km}$, a mere $1800 \mathrm{Km}$ outside the photon sphere.

Although a point mass can rest comfortably in that orbit, a mega-structure is subject to tidal forces which are of considerable concern in such intense gravity. We need to calculate this force to ensure it doesn't tear the structure (or its inhabitants) apart.

The natural way to design a structure to minimize tidal forces is to extend it along an arc of the circular orbit (see Fig. 2:

Figure 2: Tidal forces for orbiting structure

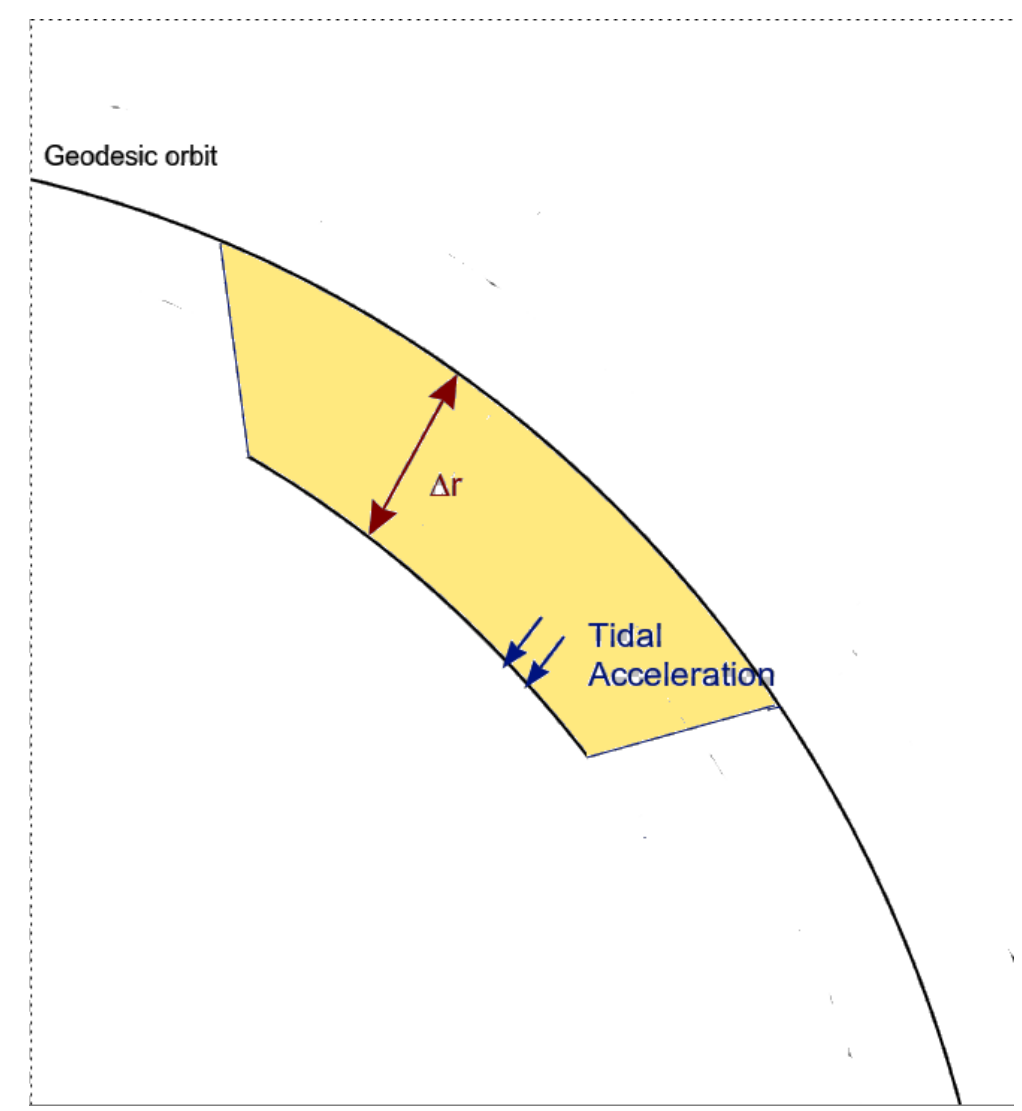

We will set the maximum tidal acceleration we can tolerate and calculate the corresponding 'height' (radial extent) that it allows. This height is given by,

$$
\Delta r=\frac{A_{t} r^{3}}{3 G M} \text { where } A_{t} \text { is tidal acceleration. [A 7.1] }
$$


For our example of Sag $\mathrm{A}^{*}$, we set the tidal acceleration equal to the surface gravity of Earth and evaluate :

$$
\Delta r=\frac{9.8\left(18 \times 10^{9}\right)^{3}}{3\left(6.67 \times 10^{-11}\right)\left(8.15 \times 10^{36}\right)}=35 \mathrm{Km}
$$

35 kilometers seems like quite a generous high ceiling - but we must take care to account for the dilation of space within the megastructure. In order to maintain the constancy of local measurements of $\boldsymbol{c}$, space must dilate in proportion to the dilation of time. Given our $\gamma=100$, the height is measured to be 350 meters within the structure. We can double this to 700 meters in order to create a two-layer structure as shown in Fig. 3.

Figure 3: Doubling the height and balancing the tidal force.

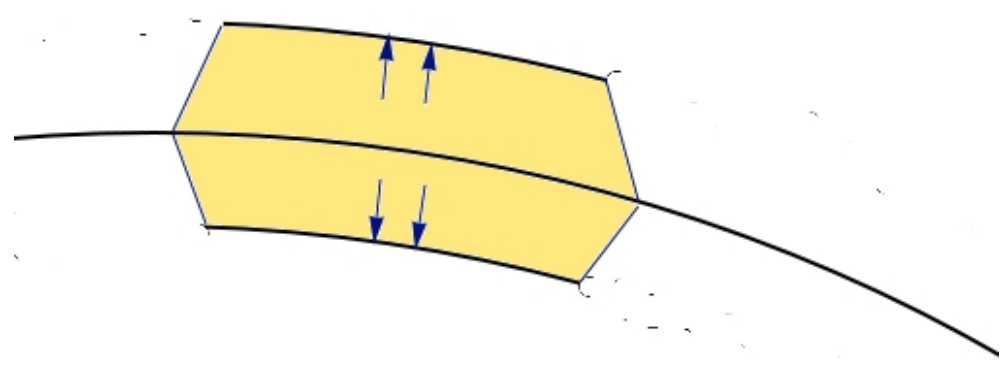

This straddling of the orbit is necessary not only to maximize radial height; the tidal forces must be balanced to hold orbit. Interestingly, these tidal forces are seen to serve a positive role providing the inhabitants with a comfortable sense of gravity.

While 700 meters is admittedly a bit cramped for a civilization-bearing megastructure, this restriction in a single dimension does not appear fatal to our hypothesis. We note that the structure can extend without bound along the orbital path, yielding surface area greater than Earth's.

We caution that our calculation of the tidal force neglects relativistic effects; it is only intended as an illustrative approximation. For a full relativistic treatment see, for example, [Y Yri (2017)]

The other force to be considered is the Coriolis effect. Within the structure, a massive ball suspended on a string will be seen to rotate once every orbital period. We need to calculate this period to ensure that the Coriolis effect isn't hazardous to such delicate biological structures as ourselves.

As seen from afar, the orbital period for any dilation is found by applying Kepler's Third Law to the radius given in Eq. (2) [see, e.g., [L Lambourne (2009)] : 


$$
\text { orbital period }=\Omega / 2 \pi=\frac{1}{2 \pi} \sqrt{\frac{G M}{\left[r_{p h}\left(1+\frac{1}{3 \gamma^{2}}\right)\right]^{3}}}
$$

Again we need to correct for local space-time dimensions; within the satellite, time dilation reduces the observed local period to period $/ \gamma$. For our example of Sag $\mathrm{A}^{*}$ and $\gamma=100$, the local orbital period is found to be 6.489 seconds.

Thus our ball suspended by string would rotate once every 6.489 seconds. While this effect is far from negligible, it also does not appear a fatal flaw to our hypothesis. The plot below shows the Coriolis effect is intense only for large time dilations. 
Figure 4

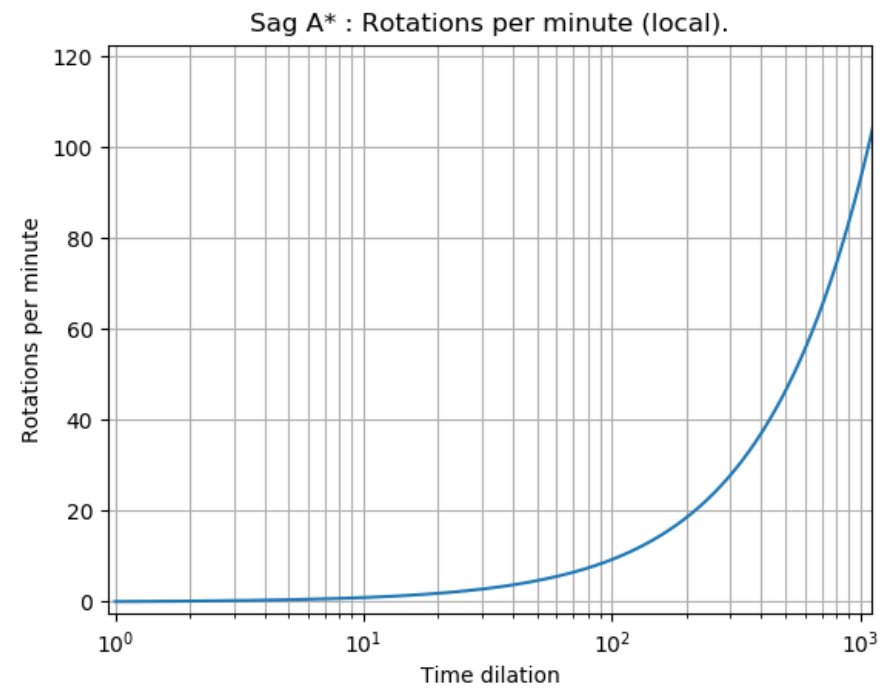

The other challenges of occupying such a hostile region of space, enduring the onslaught of both inbound matter and outbound radiation, certainly appear daunting. But these hazards are perhaps no more difficult than Apollo 11's defiance of heat, gravity, and vacuum would have seemed to the Wright brothers.

These hazards might be mitigated by an orbit which is tilted with respect to the galactic plane in order to minimize interaction with matter inbound from the accretion disk. (Such a non equatorial orbit could make it easier to detect the structure; EM emissions would acquire a vertical polarization which stands apart from other sources in the galactic plane.)

There is also recent evidence to support that Sgr A* may be unusually 'tame' due its magnetic field which tends to hold the accretion disk a safe distance from the ISCO. [Bell et al.(2020)]

If we posit that such a red-satellite exists and is producing radio emissions, either intentionally or incidentally, we can make certain inferences about that signal. The prospects of detecting a radio signal emitted so close to Sgr $A^{*}$ do seem bleak due to the radio noise Sgr A* produces across a broad spectrum. However, the unique orbit of a red-frame satellite would lend a distinct signature to any radio signal it emits. We look to the combined effects of doppler beaming and gravitational lensing.

\section{EM signature of an orbiter below the ISCO}

Consider again a red-satellite of a $\mathrm{SMBH}$ which is emitting some EM signal that is observed at $r=\infty$. We seek criteria that differentiate the observed signal of a red-satellite from those of natural (unguided) orbiters above the ISCO.

We shall insist these criteria be independent of the black hole mass (M) about which we may be 
uncertain, even for Sag A*.

For a Schwartzschild black hole of any mass, the (local, proper) orbital speed of a test particle in stable orbit in the ISCO is fixed at $v_{o}=1 / 2$ (see Eq.(3)) At this speed, the doppler shift will vary between some minimum $F_{\text {low }}$ as the satellite recedes along the line-of-sight and $F_{\text {high }}=3 F_{\text {low }}$ as it directly approaches.

A red-frame satellite in unstable orbit below the ISCO will have a higher orbital velocity which induces a doppler shift cycling outside the range of $\left[F_{\text {low }}, 3 F_{\text {low }}\right]$.

This feature alone, of course, is insufficient to identify a red-frame technosignature. To sharpen our search criteria, we examine more fully the effect of relativistic doppler beaming :

It is a little counter-intuitive that, despite the red-frame's perilous proximity to the SMBH, most of the time-dilation is due to motion and not gravity. Since the radius (for any time dilation) must be outside the photon sphere, this bounds the gravitational red-shift :

$$
\text { Since } r>3 M \text {, gravitational red-shift } \gamma_{g}=1 / \sqrt{g_{t t}}=1 / \sqrt{1-\frac{2 M}{r}}<\sqrt{3}=1.732 \text {. }
$$

Any time dilation beyond 1.732 is induced by orbital motion close to the speed of light. For any circular Keplerian orbit about a Schwartzschild $\mathrm{BH}$, the local orbital velocity $v_{o}$ is given by [see e.g., Opatrny et al., (2017)]:

$$
v_{o}=\sqrt{\frac{M}{r-2 M}}
$$

Subtituting for $r$ via Eq. (2) gives,

$$
v_{o}=\sqrt{\frac{\gamma^{2}-1}{\gamma^{2}+2}}
$$

For our example satellite with $\gamma=100$, the orbital velocity is $v_{o}=0.99985$.

At such speeds, the effect of relativistic doppler beaming becomes far more pronounced than for a body in natural orbit. 


\section{$5.1 \quad$ Doppler beaming}

For a light source moving at speed $v$ with emission angle $\theta_{e m}$ with respect to the direction of motion, the frequency observed by a relatively static $(\mathrm{v}=0)$ receiver $F_{o}$ varies from the source frequency $F_{s}$ according to (see, e.g., [Hartle 2008]] :

$$
F_{o}\left(v, \theta_{e m}\right)=\frac{F_{s} \sqrt{1-v^{2}}}{1-v \cos \left(\theta_{e m}\right)}
$$

Substituting for $v$ via Eq. (4) gives :

$$
F_{o}\left(\gamma, \theta_{e m}\right)=\frac{F_{s}}{\gamma-\cos \left(\theta_{e m}\right) \sqrt{\gamma^{2}-1}}
$$

As velocity approaches the speed of light (and $\gamma$ increases), this beams almost all the signal energy into an 'optic boom' in the form of a narrowing cone as illustrated in Fig 5 :

Figure 5: Illustration of doppler beaming close to $\boldsymbol{c}$

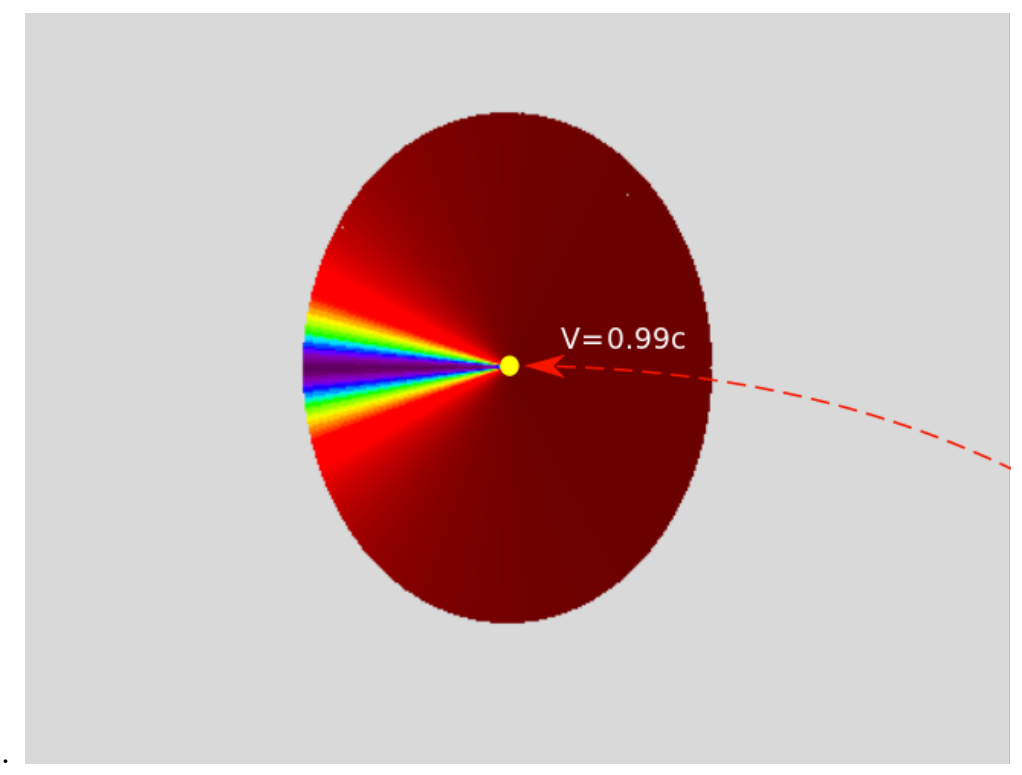

recalculate this For our example of $\gamma=100,67 \%$ of the energy is contained within 2 degrees of the sight-line [A2]. As our emitter revolves through its orbit, it is clear that if we negate the effect of gravitational lensing (e.g., by replacing gravity with thrust) the doppler shift will be distantly observed as a series of sharp pulses which are nearly symmetric in time. 
In the following section we will see how gravitational lensing breaks that symmetry.

\subsection{Gravitational lensing}

Consider a satellite in circular geodesic orbit around a Schwartschild BH outside the photon sphere (but not necessarily the ISCO.) The satellite emits a signal which is constant, monochromatic and isotropic. The emission angle is taken with respect to a radial line passing through the emitter (Fig $6)$ :

\section{Figure 6}
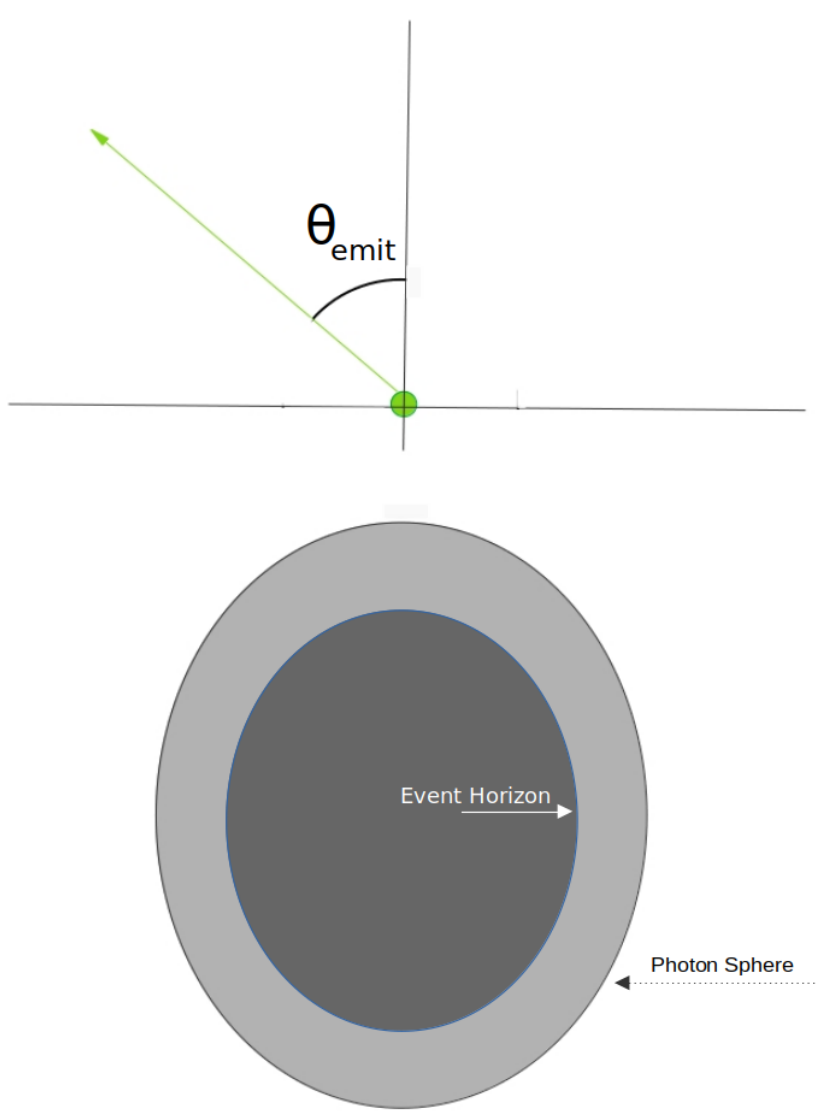

The path of a photon in any gravitational field is fully described by the set of differential equations, 


$$
g_{\mu v} d x^{\mu} d x^{v}=0, \quad \frac{d^{2} x^{\mu}}{d s^{2}}+\Gamma_{\mu}^{v \sigma} \frac{d x^{v}}{d s} \frac{d x^{\sigma}}{d s}=0
$$

We set $g_{\mu v}$ to the Schwartschild metric, and restrict our attention to scattering paths (i.e., only those observable at $r=\infty$.) This system is readily solvable by segmenting into two cases. (Our treatment of the photon path takes much from [De Falco 2016] and

Case 1: Where $\theta_{e m}<=\pi / 2$ (as in Fig 6 ), the photon strictly recedes from the $\mathrm{BH}$ as it moves along its path. This monotonicity enables the solution:

ter.)

$$
\begin{aligned}
& \Omega_{1}\left(\theta_{e m}, r\right)=\int \frac{d r}{\sqrt{1 / Q(r)}} \text { where } Q\left(\theta_{e m}, r\right)=b\left(\theta_{e m}\right)^{2} r^{4}-r^{2}+2 M r \\
& \text { and where } b\left(\theta_{e m}\right):=\frac{r}{\sqrt{1-r_{s} / r}} \sin \left(\theta_{e m}\right) . \quad\left(b\left(\theta_{e m}\right)\right. \text { is termed the impact parame- }
\end{aligned}
$$

This integral has a solution which is analytic in terms of EllipticF, the incomplete elliptical integral of the first kind:

$$
\begin{aligned}
& \Omega_{1}\left(\theta_{e m}, r\right)=\frac{2}{r} \text { EllipticF } {\left[\arcsin \left(\sqrt{\frac{q_{3}-1 / r}{q_{3}-q_{2}}}\right), \frac{q_{3}-q_{2}}{q_{3}-q_{1}}\right] } \\
& \times \sqrt{\left(q_{1} r-1\right)\left(q_{2} r-1\right)\left(q_{3} r-1\right)\left(b\left(\theta_{e m}\right)^{2}-r+2\right)} .
\end{aligned}
$$

where $q_{1}, q_{2}, q_{3}$ are the roots of $x^{3}-x^{2}+\frac{\sin ^{2} \theta_{e m}}{3}=0$. These roots are ordered such that $\operatorname{Im}\left[q_{1}\right]=0$ and $\operatorname{Re}\left[q_{2}\right]>\operatorname{Re}\left[q_{3}\right]$. 
Case 2: $\left|\theta_{e m}\right|>\pi / 2$

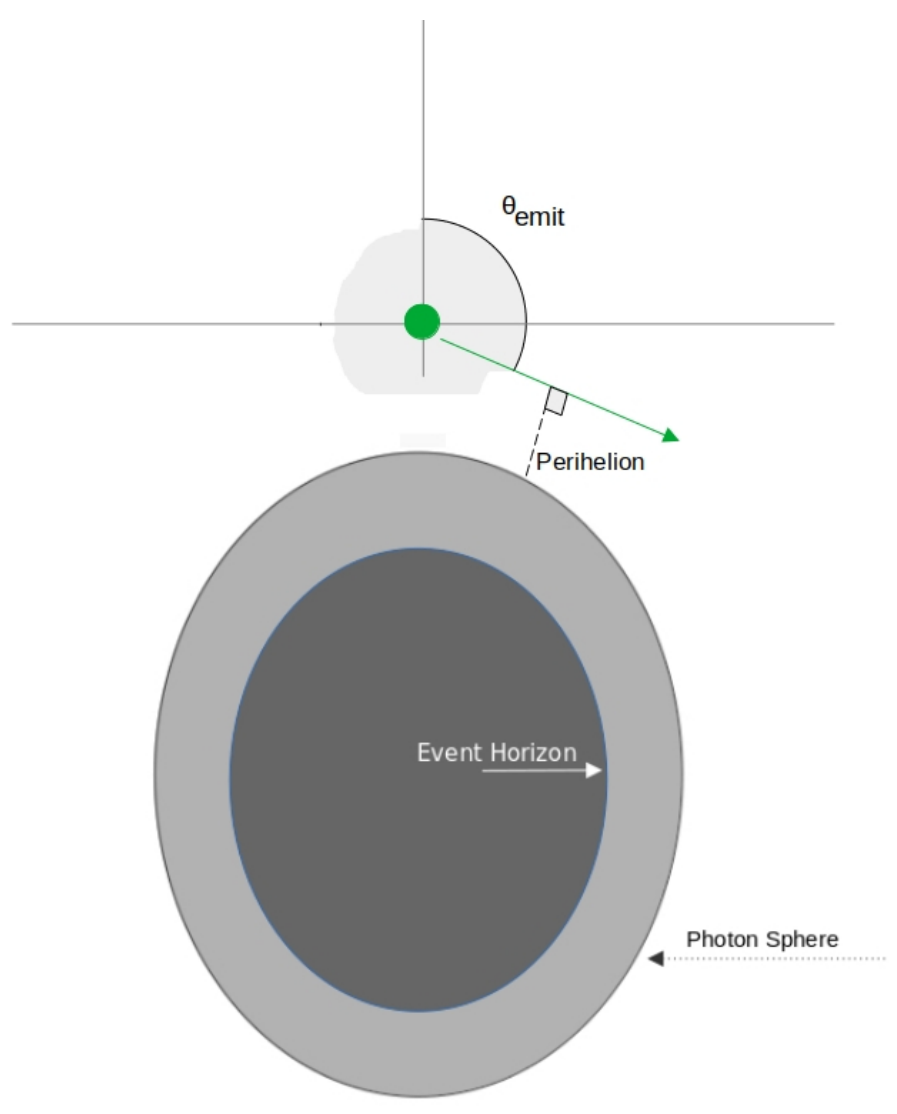

At this emission angle, the photon first approaches the BH before flying off. We find the point of closest approach $\mathrm{P}$ (perihelion). From $\mathrm{P}$ onward, the path gains distance monotonically and is thus amenable to the solution in Case 1.

The value of $\mathrm{P}$ is found as a function of $r$ and $\theta_{e m}$ by (see, e.g., [Bisnovatyi-Kogan 2008]]:

$$
\begin{aligned}
& P\left(\theta_{e m}, r\right)=\frac{a}{3^{2 / 3}}+\frac{b^{2}}{3^{1 / 3} a} \\
& \text { where } \mathrm{b}=\frac{r}{\sqrt{1-r_{s} / r}} \sin \left(\theta_{e m}\right) \\
& \text { and we abbreviate } a=\left[-(3 b)^{2}+\sqrt{3} b^{2} \sqrt{3^{3}-b^{2}}\right]^{1 / 3}
\end{aligned}
$$


The maximum emission angle $\theta_{\text {Max }}$ that avoids being trapped by the photon sphere is : [De Falco 2014

$$
\theta_{\text {Max }}=\pi-\arcsin \left[\frac{3}{r} \sqrt{\frac{3}{1-2 / r}}\right]
$$

We can now write the general formula for all escaping paths from the emitter:

$$
\Omega\left(\theta_{\text {em }}, r\right)=\left\{\begin{array}{cl}
\Omega_{1}\left(\theta_{e m}, r\right) & \left|\theta_{\text {em }}\right|<=\pi / 2 \\
2 \Omega_{1}\left(\theta_{\text {em }}, r\right)-\Omega_{1}\left(\pi / 2, P\left[\theta_{e m}, r\right]\right) & \pi / 2<\left|\theta_{e m}\right|<\theta_{\text {Max }}
\end{array}\right.
$$

(7)

Where $\left|\Omega\left(\theta_{\text {em }}, r\right)\right|>2 \pi$, the photon takes at least one round trip around the BH (i.e., has non zero winding number.) before arriving at angle $\left[\Omega\left(\theta_{\text {em }}, r\right)\right.$ мор $\left.2 \pi\right]$

For our emitter of $\gamma=100$, we plot the light paths over a select range of possible emission angles. Each path is colored according to its Doppler shift as in Fig 5 :

Figure 7

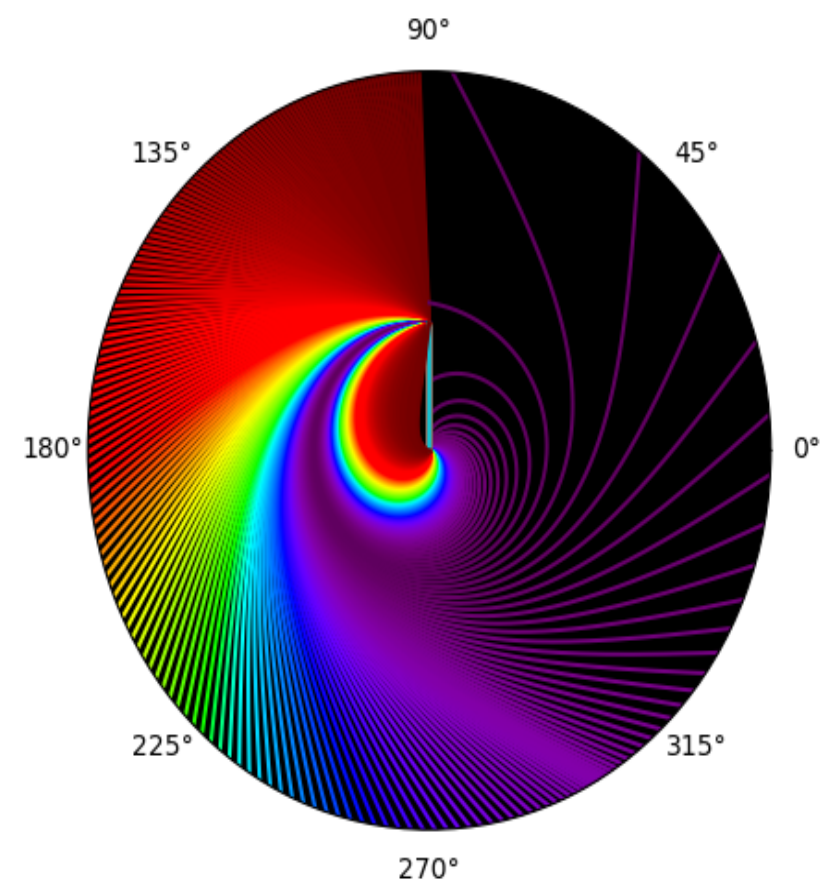


As the emitter revolves, we see that gravitational lensing breaks the time-symmetry of the outgoing signal. A distant observer will again see a series of pulses, but each pulse will appear at high frequency and then decline. (The ascending half of the signal has mostly fallen into the BH. This 'sad trombone' effect is also a salient feature of Repeating Fast Radio Bursts. [Rajabi 2020] [Pearlman 2022]

We shall make precise this rough observation and calculate the observed signal at $r=\infty$. For the remainder of this section, we shall take the emitter to be a 'red satellite' where $\gamma=100$. (The radius is given by $\mathrm{Eq}(2)$ and the velocity by $\mathrm{Eq}(4)$.)

Define $\Omega_{\infty}\left(\theta_{e m}\right):=\lim _{r \rightarrow \infty} \Omega(\theta, r)$. (For our purposes of computation, $\Omega\left(\theta_{e m}, 10^{10}\right) \approx \Omega_{\infty}\left(\theta_{e m}\right)$

We plot $\Omega_{\infty}(\theta)$ below (note the smoothness of $\Omega_{\infty}$ despite its piecewise definition) :

Figure 8: Final outbound angle $\Omega_{\infty}(\theta)$ versus angle of emission $\theta$

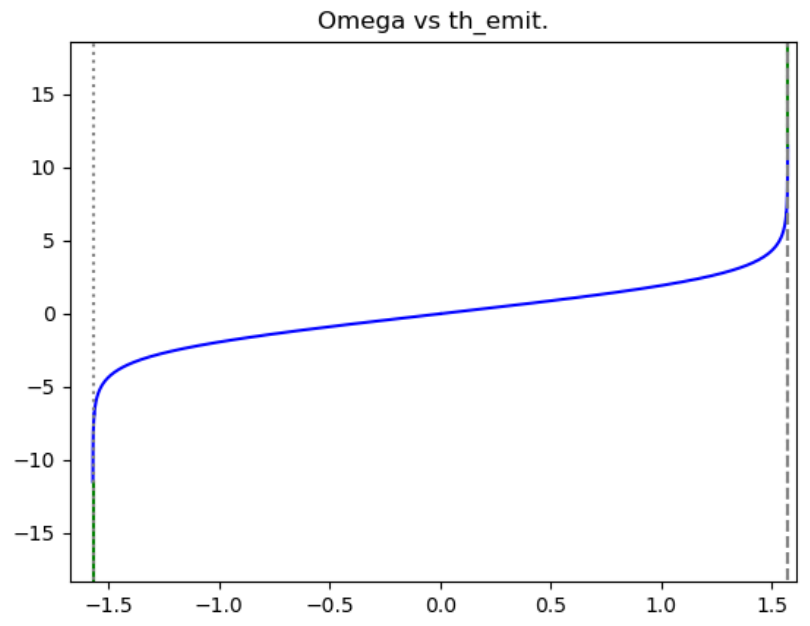


Figure 9: Final outbound angle $\Omega_{\infty}(\theta)$ versus angle of emission $\theta$

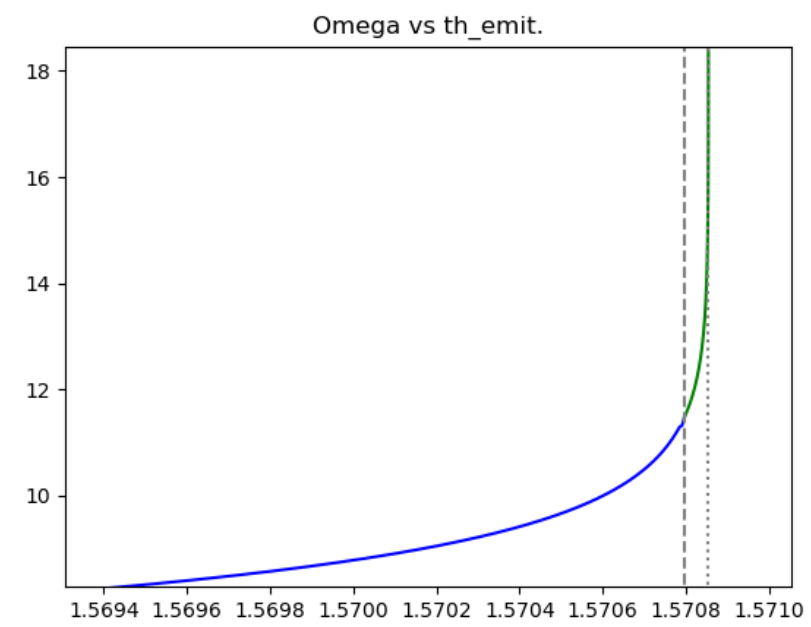

Let us now set the emitter revolving at orbital angular velocity $\omega$. By rotational symmetry, the photon path ${ }^{5}$ originating from $\theta_{\text {emit }}$ will distantly terminate at angle $\left[\Omega\left(\theta_{\text {emit }}\right)+\omega t\right]$ мор $2 \pi$. If we set our perspective counter-rotating by $-\omega$, the emitter (as before) appears static while the observer circles the BH. It becomes clear that for a single observer at fixed angle, the path originating from $\theta_{\text {em }}$ sweeps across the distant observer at time

$$
t\left(\theta_{e m}\right)=\frac{\left[k_{0}-\Omega\left(\theta_{e m}\right)\right] \text { мор } 2 \pi}{\omega} \text { where } k_{0} \text { is an initial offset due to observer angle. }
$$

We can use this relation to plot the frequency curve for a distant observer. If we resist the temptation to invert Eq. 7 , we can proceed more directly by treating $F\left(\gamma, \theta_{e m}\right)$ and $t\left(\theta_{e m}\right)$ as parametric functions of $\theta_{e m}$. By sweeping a sufficiently inclusive set of (hidden) $\theta_{e m}$ values, we plot $\mathrm{F}$ vs time for a distant observer :

\footnotetext{
${ }^{5}$ The travel time of the photon is not of interest; we are only concerned with the rotational motion of the light path due to orbital motion.
} 
Figure 10: Observed Frequency over Time

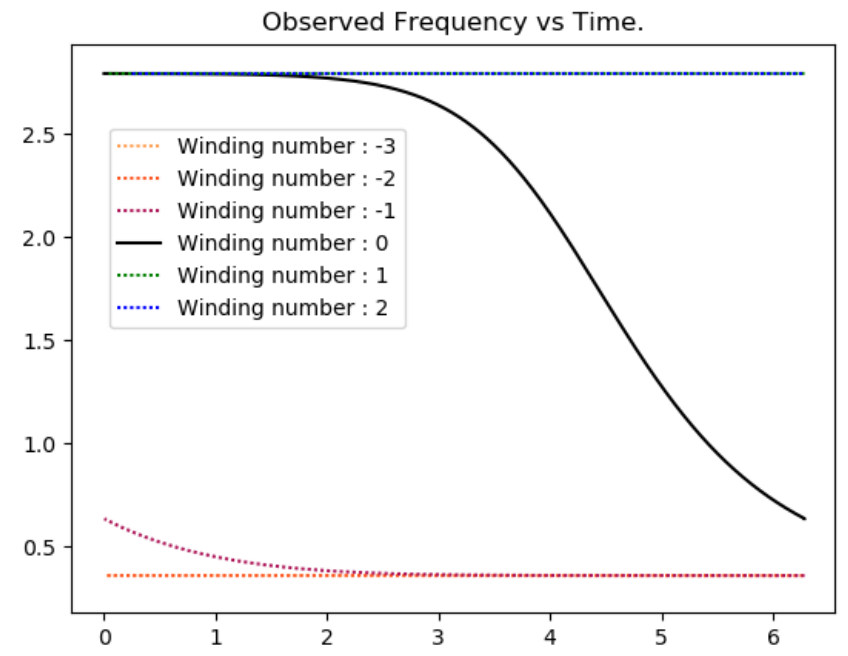

Figure 11: Same graph as Fig 9 zoomed in 2500x to show winding numbers $w=1,2$

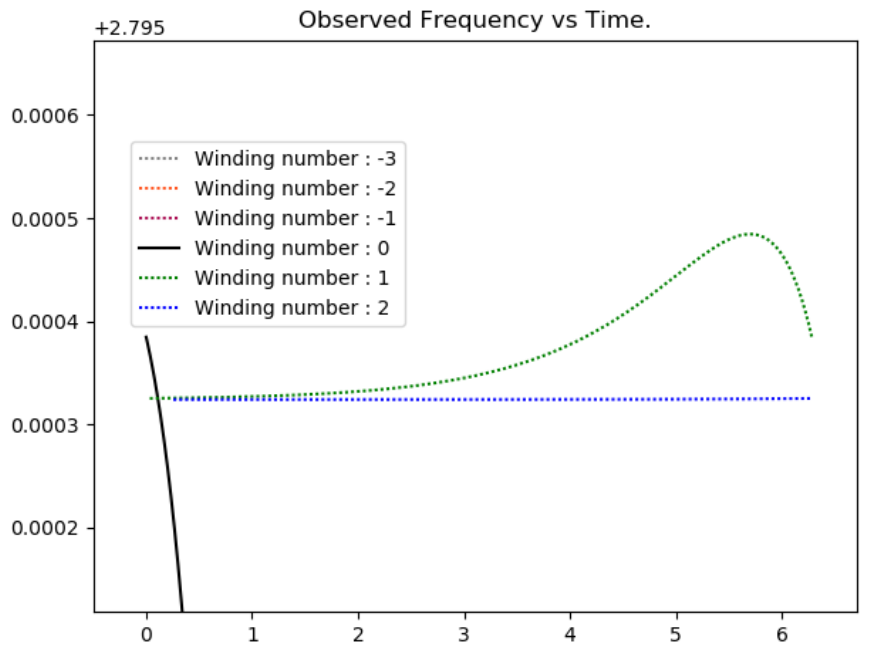

We see how the observer is impacted by the superposition of signals along paths of every winding number. It is only the most direct $(\mathrm{w}=0,-1)$ paths, however, that show a significant frequency delta which manifests as a regular cascade of frequency drops. The indirect $(w \neq 0,-1)$ paths deliver 
frequency curves that are nearly flat and nearly indistinct.

We take note that, unlike the near-symmetric doppler wobble of a satellite in the weak field, the frequency curve of a red-satellite sheds its symmetry to become nearly monotonic. 
For our isotropic radiator, the signal Amplitude is the inverse of the optical magnification of the gravitational 'lense' :

$$
A\left(\theta_{e m}\right)=\frac{d \theta_{e m}}{d \Omega_{\infty}\left(\theta_{e m}\right)}=1 / \Omega_{\infty}^{\prime}\left(\theta_{e m}\right)
$$

The derivative of $\Omega_{\infty}$ can be calculated symbolically by Eq. 7, but the result is lengthy and complex. For our purposes it suffices to compute $\Omega_{\infty}^{\prime}$ numerically.

Energy flux density (EFD) is given by, $E\left(\theta_{e m}\right)=F\left(\pi / 2-\theta_{e m}\right) A\left(\theta_{e m}\right)^{2}$. To plot EFD over time, as before we parameterise by $\theta_{e m}$ in order to plot $E\left(\theta_{e m}\right)$ against time $t\left(\theta_{e m}\right)$ (from Eq. 8)

Figure 12: Energy flux density over time

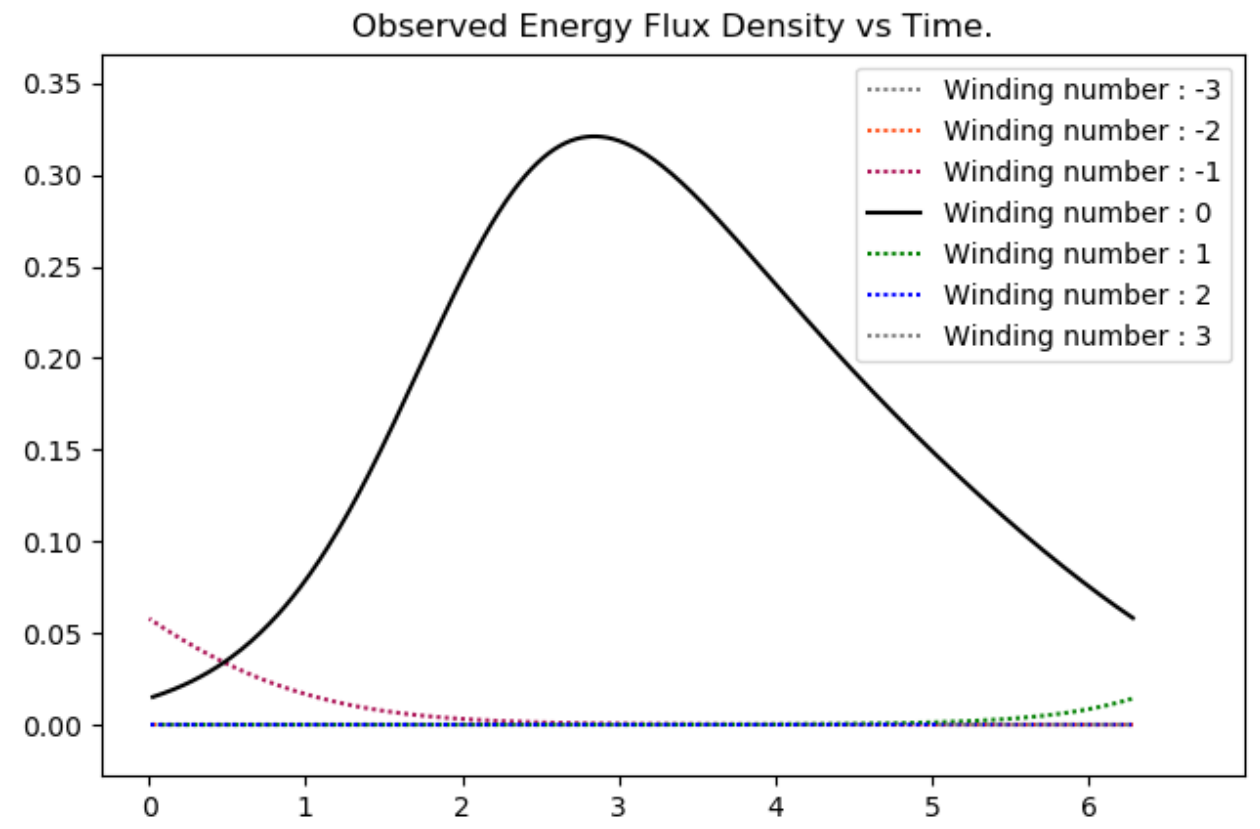


Figure 13: Same as Fig 11, zoomed in 2,000,000 X to show signal from higher winding paths.

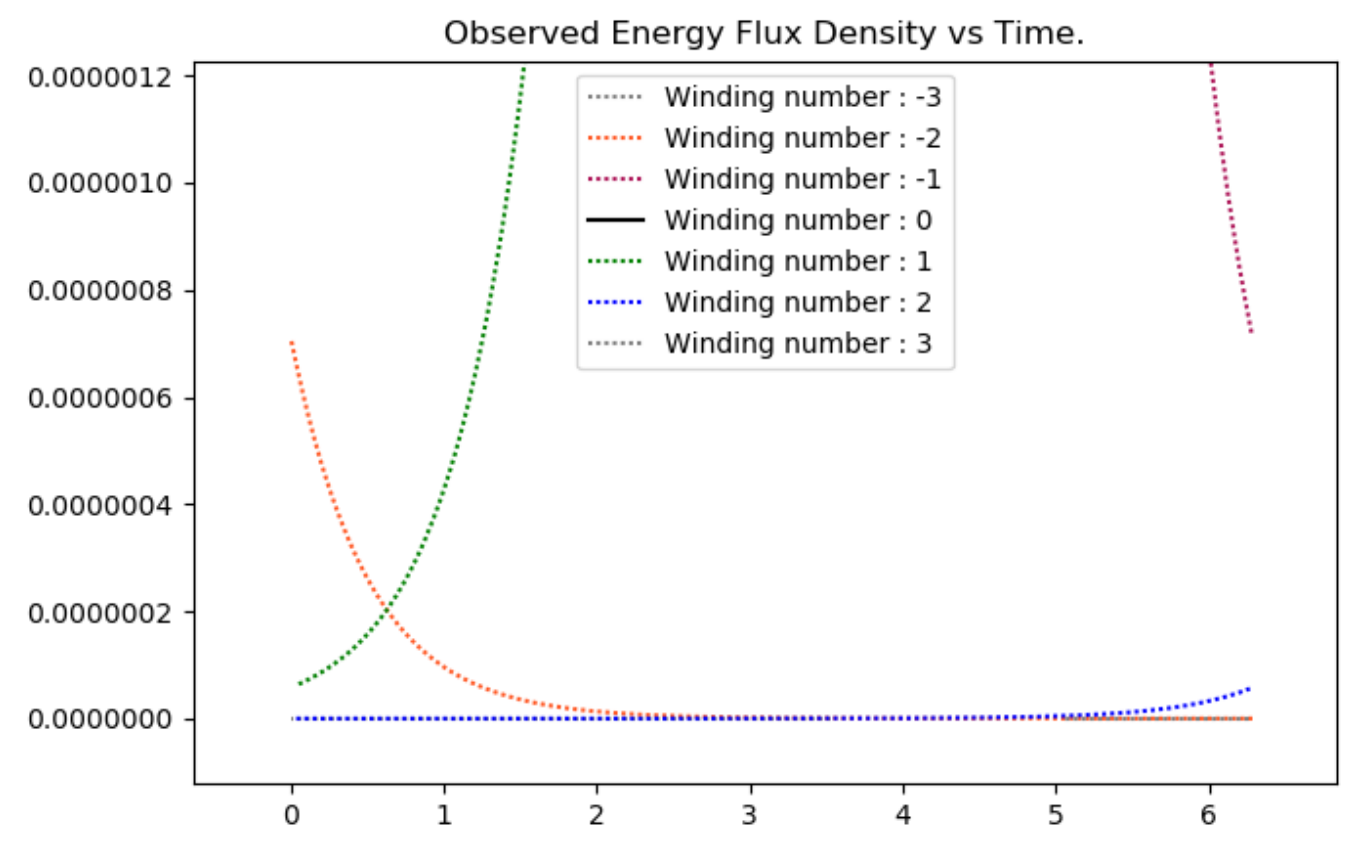

The graph shows that winding numbers outside the range $-1,0,1$ contribute very little flux to the signal. For many practical purposes such as ours, these paths can be ignored. 
Figure 14: Energy Flux Density overlayed with Frequency. Frequency shown in grey and also as flux curve color.

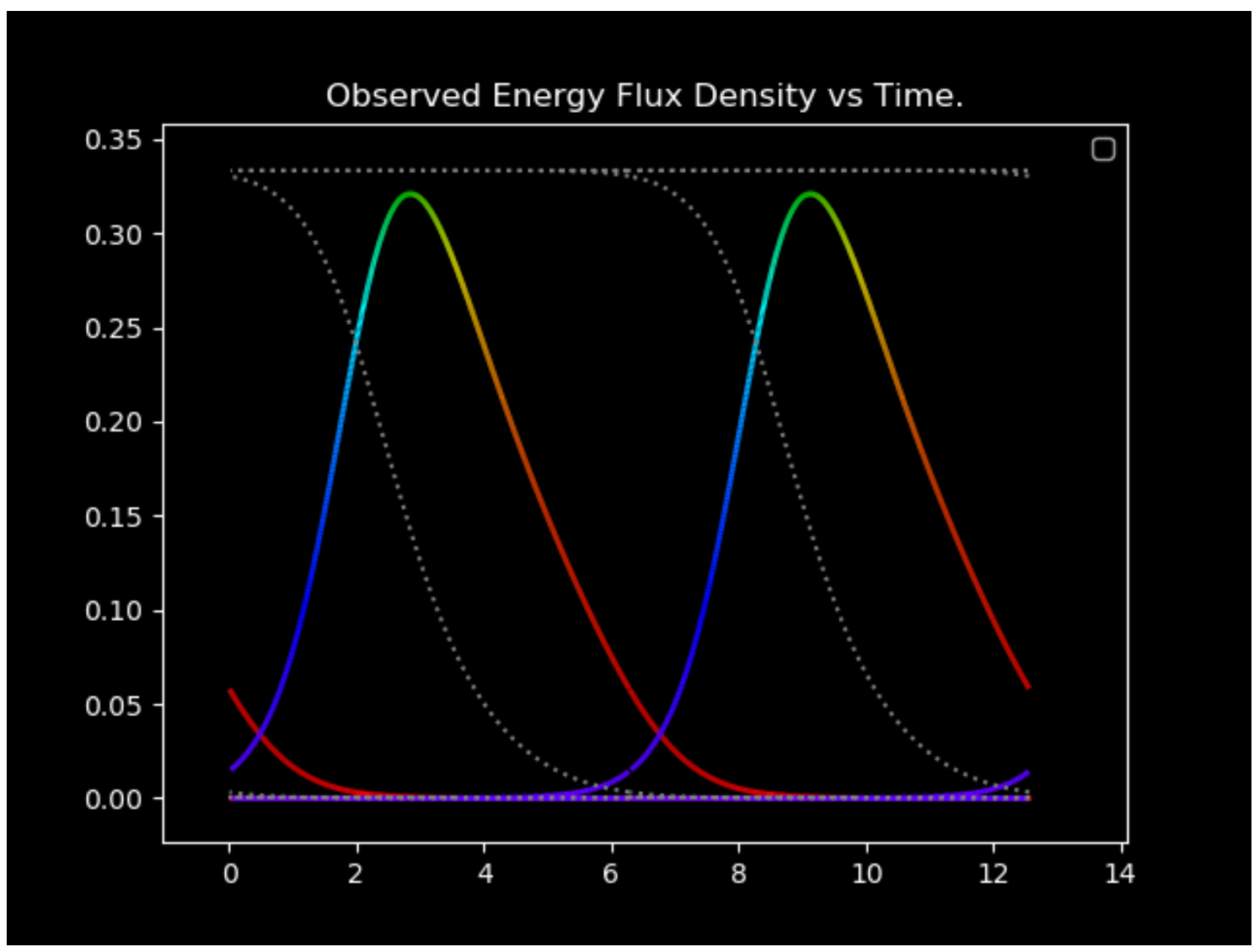

If we overlay frequency and flux density for a couple of orbital periods (Fig. 14), we see the general character of each cycle : A pulse appears dimly at a high, constant frequency. It gets rapidly brighter as frequency begins an accelerating decline. The decline peaks just before peak flux density. Finally, both color and flux density fade off.

In the background, there is a weak, sustained signal at the upper and lower frequency range of the signal. These are formed by paths with higher winding numbers, whose frequency curves are nearly indiscernibly close. (A negative winding number produces a maximum frequency, a positive w a minimum.) 
For a Schwartzschild BH, a periodic series of pulses of this form is indicative of a guided orbiter near the speed of light just outside the photon sphere.

\subsubsection{Invariant property}

We seek a property of doppler beaming that is invariant under gravitational lensing. This leads to a simple and robust test that helps determine if an observed signal is induced by a circular orbiter.

For a Kerr black hole, and a distant observer coplanar with a radiator's circular (equatorial) orbit, this relation holds :

$\left(c_{1} \dot{\boldsymbol{F}}(t) / E(t)-1\right)^{2}+\left(c_{2} F(t)-1\right)^{2}=1$

where $\mathrm{F}(\mathrm{t})$ is observed frequency, $\dot{\boldsymbol{F}}(t):=d F(t) / d t$ is observed frequency drift , $E(t):=F(t) A(t)^{2}$ where $A(t)$ is observed signal amplitude, $\mathrm{c} 1$ and $\mathrm{c} 2$ are constants.

So $\dot{\boldsymbol{F}}(t) / E(t)$ vs $F(t)$ forms a circle for suitable c1,c2.

\section{Proof(ish) :}

So we agree that up to the Kerr case, for an emission angle of $\theta_{\infty}$ as observed in the distant receiver's frame :

1) $F_{\infty}\left(\theta_{\infty}\right)=F_{s} \operatorname{Dop}_{\infty}\left(\theta_{\infty}, v\right) / \gamma_{g}$

$$
\begin{aligned}
& \text { where } \operatorname{Dop}_{\infty}\left(\theta_{\infty}, v\right):=\frac{1}{\gamma_{v}\left(1+v \cos \left(\theta_{\infty}\right)\right)} \\
& \gamma_{v}:=1 /\left(1-v^{2}\right) \text { and } \gamma_{g} \text { is gravitational time dilation. }
\end{aligned}
$$

That is - just the special relativistic doppler effect scaled by gravitational time dilation $\gamma_{g}$. In what follows, we appear to take an ill-fated interest in the emission angle, which is obscured behind the black hole parameters. But our scheme is to tease some information out of various relations to $\theta$ before we knock that variable out of our final form.

We'd like to use the fact the emitter is isotropic.

So we need to consider the emission angle $\vartheta_{\text {local }}$ as measured in the local frame of the emitter.

Happily, in his 1905 paper Einstein calculated the same $\operatorname{Dop}()$ as in 1) but in terms of this local angle $\vartheta_{\text {local }}$ (see section 7 of his 1905 paper)

2) $\operatorname{Dop}_{\infty}\left(\theta_{\infty}, v\right)=\operatorname{Dop}_{\text {local }}\left(\vartheta_{\text {local }}, v\right)=\gamma_{v}\left(1-v \sin \left(\vartheta_{\text {local }}\right)\right)$

where only $\vartheta_{\text {local }}$ is in the emitter's local frame ( $\mathrm{v}$ and $\mathrm{t}$ are both in the observer frame.) Einstein doesn't leave the frames mixed like this, in the next paragraph he gives the 'angle aberration' formula to bridge the gap. But it happens to be of use to us in this form.

Now let's see what happens to a tiny arc of emission angles on their trip outward. The arc of emission angles in the range $\left[\vartheta_{\text {local }}, \vartheta_{\text {local }}+d \vartheta_{\text {local }}\right]$ light up with the same frequency and amplitude (since the emitter is isotropic).

We define angular energy density $E$ at angle $\rho, E(\rho):=F(\rho) A(\rho)^{2}$, so that the total energy of an arc is given by ArcEnergy $[a, b]=\int_{a}^{b} F(\rho) A^{2}(\rho) d \rho$. (Do I need a pf here that ArcEnergy is 
conserved as $r \rightarrow \infty$ ?) Then by definition the ArcEnergy of the infinitesimal arc is

ArcEnergy $\left[\vartheta_{\text {local }}, \vartheta_{\text {local }}+d \vartheta_{\text {local }}\right]=F\left(\vartheta_{\text {local }}\right) A^{2}\left(\vartheta_{\text {local }}\right) d \vartheta_{\text {local }}$

$=F_{k} A_{k}^{2} d \vartheta_{\text {local }}=E_{k} d \vartheta_{\text {local }}$ since the emitter is isotropic.

The light from this arc ends up in $\left[\theta_{\infty}, \theta_{\infty}+d \theta_{\infty}\right]$ (in the coordinate frame) as $r \rightarrow \infty$. Since energy is conserved, the total energy must stretch along the distant arc, reducing the angular energy density, . (This is optical amplification of the 'lense'.) That is,

3) $E_{\infty}\left(\theta_{\infty}\right)=E_{\text {local }}\left(\vartheta_{\text {local }}\right) \frac{d \vartheta_{\text {local }}}{d \theta_{\infty}}$ Let's put a pin in that.

Since the emitter is revolving in its coplanar orbit, the distant observer can associate $d \theta_{\infty}$ with a duration of time $d t$ (since other $\theta_{\infty}$ angles will rotate into view). Thus :

4) $\frac{d F_{\infty}\left(\theta_{\infty}\right)}{d \theta_{\infty}}=\omega \frac{d F_{\infty}(t)}{d t}$ where $\omega$ is the orbital angular velocity of the orbiter (in the observer/coordinate frame.)

We note that both $F_{\infty}(t)$ and $d F_{\infty}(t) / d t$ are observable. We'll write $\dot{\mathbf{F}}:=\frac{d F}{d t}$

OK, unpin : We're going to use the amplification in 3) to sort of bridge the two coordinate frames :

Recalling 1),

$$
F_{\infty}\left(\theta_{\infty}\right)=D_{\infty}\left(\theta_{\infty}\right) / \gamma_{g}
$$

We're going to mix coord frames here as we substitute Einstein's local doppler formula,

$$
F_{\infty}\left(\theta_{\infty}\right)=D_{\text {local }}\left(\vartheta_{\text {local }}\right) / \gamma_{g}
$$

Take $D / d \theta_{\infty}$ of both sides,

$$
\frac{d F_{\infty}\left(\theta_{\infty}\right)}{d \theta_{\infty}}=\frac{d D_{\text {local }}\left(\vartheta_{\text {local }}\right)}{d \theta_{\infty}} / \gamma_{g}
$$

Set up the Chain rule,

$$
=\frac{d D_{\text {local }}\left(\vartheta_{\text {local }}\right)}{d \vartheta_{\text {local }}} \frac{d \vartheta_{\text {local }}}{d \theta_{\infty}} / \gamma_{g}
$$

Subst for $\frac{d \vartheta_{\text {local }}}{d \theta_{\infty}}$ from 3$)$,

5) $=\frac{d D_{\text {local }}\left(\vartheta_{\text {local }}\right)}{d \vartheta_{\text {local }}} \frac{E\left(\theta_{\infty}\right)}{E_{k}} / \gamma_{g}$

Differentiating Eq 2) gives,

6) $\quad \frac{d D_{\infty}\left(\vartheta_{\text {local }}\right)}{d \vartheta_{\text {local }}}=\gamma_{v}\left(1-v \cos \left(\vartheta_{\text {local }}\right)\right)$ 
Subst back into 5) to get finally,

7) $\frac{d F_{\infty}\left(\theta_{\infty}\right)}{d \theta_{\infty}}=\frac{\gamma_{v}}{\gamma_{g}}\left(1-v \cos \left(\vartheta_{\text {local }}\right)\right) E\left(\theta_{\infty}\right) / E_{k}$

Applying the trig indentity $\cos ^{2}+\sin ^{2}=1$ to 1 ) and 7) knocks out the variable $\vartheta_{\text {local }}$ and yields the relation,

$\left(c_{1} \dot{\boldsymbol{F}}(t) / E(t)-1\right)^{2}+\left(c_{2} F(t)-1\right)^{2}=1$

where $\mathrm{c} 1$ and $\mathrm{c} 2$ absorb constants (including $v$ ) along the way. So - with the right scaling, a plot of $\dot{\boldsymbol{F}} / E$ vs $F$ becomes a circle.

This is a necessary (but insufficient) condition for observations of an emitter in circular orbit. This serves as a simple test to discard signals not induced by the rotating doppler beam of a circular orbiter . (The values $\frac{d F}{d t}, F$, and $A$ are observed at two points; and one solves for $k$ and $\gamma$. A third observation is then compared against its predicted value. Should the signal survive this test, the experimenter can then proceed to curve-fit the data against a full lensing model, such as the one we describe in the previous section for the Schwartzschild case. )

Since we made no appeals to symmetry, this test is not constrained to the Schwartzschild case; it is equally valid for a Kerr-Newman Black Hole. We only require an isotropic emitter in a circular orbit coplanar with the observer. Then Eq 9 holds for any differentiable lensing function. 


\section{Discussion and Conclusions}

We've shown that it is possible for an ETI to overcome the obstacle imposed by the speed of light by permanently occupying a reference-frame where time is dilated (slowed) by orders of magnitude. One possible location of such a reference-frame is shown to be in near-orbit around a SMBH between the photon sphere and Innermost Stable Circular Orbit.

A method is proposed for detecting an artificial mega-structure in such an orbit: EM radiation from such a source is shown to beam into pulses of declining frequency according to Eq. (7), Eq (8) and Eq (4).

The signal bears some semblance to the cyclic 'sad trombone' of Repeating Fast Radio Bursts. However, it lacks the brief intensity characteristic of an FRB. ${ }^{6}$ The signal also lacks the complex quasiperiodic cycles exhibited by RFRBs.

Our assumption of a Schwartzschild black hole neglects the role of black hole angular momentum and electric charge. It remains to more fully develop this line of reasoning using the Kerr-Newman metric. It also remains to consider observers that are not coplanar with the orbiter.

We have focused exclusively on a SMBH near-orbit as a means to extreme time dilation. General Relativity does, however, allow for other possibilities. We examine each of these in turn and justify setting them aside as candidates for a red-frame megastructure.

- Against Uniform Motion: One may question why we've bypassed the apparently simplest case of uniform motion near C. Here the motivating advantages of time dilation are lost as the red-frame loses the ability to maintain contact with blue-frame colonies or star systems of interest. For these purposes, a red-frame must remain confined to a region of space whose dimensions are not large relative to interstellar distances

- Against gravity alone:(I.e., stationary on the surface of a massive object.) We can quickly dispense with this possibility as a time dilation factor $=2$ would place the red-frame on a body so dense it would collapse into a black hole, pulling the red-frame contents into the event horizon.

- Against thrust alone: We might consider a circular path maintained by constant thrust rather than gravity. Here we rely on a mechanical force - thrust - to provide the inward acceleration to trace a circular path. At the speed required to achieve a 100x dilation - if we fix the radius to be that of Pluto's orbit, the red-frame experiences crushing (local, proper) centripital acceleration of the order of 100,000 G. If we fix the (local,proper) centripital force to be $1 \mathrm{G}$, the required radius balloons up to the order of 10,000 light years. This violates our requirement that the red-frame is spacially constrained. In addition, there would be a large and constant energy expenditure.

\footnotetext{
${ }^{6}$ On the other hand, since the satellite must be artificial, our assumption of isotropic emissions seems naïve. We can speculate that the signal is a directed beam forward (like headlights); perhaps in order to blast a path through plasma infalling to the $\mathrm{BH}$, or as a means of propulsion/brakes to make necessary orbital corrections.

If the emissions were a deliberate signal meant to be detected by creatures like us, the greatest peak distant signal intensity is achieved by a directed beam in the direction of the orbit.
} 
- Against stellar black holes: A stellar black hole has at most (with rare exceptions), 15 solar masses. This gives a Schwartzchild radius of $40 \mathrm{Km}$, about 1000 times smaller than that of a SMBH. This puts two critical values for a red-frame satellite out of apparently plausible range for a civilization-bearing mega-structure: For a time-dilation factor $=100$ the local (proper) orbital frequency will rise to $500 \mathrm{~Hz}$, making coriolis effects intense. The radial 'height' will constrict to about 1 meter for a tidal force of $1 \mathrm{~g}$.

A free-fall orbit near a SMBH appears the most feasible - and perhaps only - possibility. This case has virtually no sustained (theoretical) energy requirement and exerts minimal forces within the red-frame structure. Rather counter-intuitively, it is not the gravitational time dilation (whose overall contribution is small) that matters, but the more familiar gravitational attraction that serves to constrain such terrific speed to a small region of space. 7

In summary, our hypothesis suggests an explanation for the Fermi paradox : An ETI which is advanced past the point of interstellar travel will migrate at the first opportunity to a red-frame megastructure in order to escape the speed-of-light limitation. By this reasoning, the stars may only appear lifeless because advanced intelligent life has abandoned them in its migration to the galaxy's center. We therefore suggest directing some of SETI's efforts toward the center of the Milky Way and other galaxies.

\section{Appendix}

\section{[7.1] Calculation of tidal acceleration}

Let $A_{g}=$ Difference in gravitational acceleration over radial distance $\Delta r$

$$
\begin{aligned}
A_{g} & =\frac{G M}{(r-\Delta r)^{2}}-\frac{G M}{r^{2}} \\
& \approx \frac{G M}{r(r-2 \Delta r)}-\frac{G M}{r^{2}} \quad r>>\Delta r \\
& =G M / r\left[\frac{1}{r-2 \Delta r}-\frac{1}{r}\right] \quad r>>\Delta r \\
& \approx G M / r\left[\frac{2 \Delta r}{r^{2}}\right] \quad \\
& \approx \frac{2 G M}{r^{3}} \Delta r
\end{aligned}
$$

Let $A_{c}=$ Difference in centrifugal pseudo-acceleration over $\Delta r$

\footnotetext{
${ }^{7}$ We leave unaddressed the possibility of a hybrid approach where the red-frame is outside the ISCO, and kept in orbit with some assistance by constant thrust.
} 


$$
A_{c}=\frac{G M}{r^{2}} \frac{\Delta r}{r}=\frac{G M}{r^{3}} \Delta r
$$

Let $A_{t}=$ Net tidal acceleration.

$$
A_{t}=A_{g}+A_{c} \approx \frac{3 G M}{r^{3}} \Delta r
$$

$\therefore \Delta r \approx \frac{A_{t} r^{3}}{3 G M}$

\section{[7.2] Calculation of energy concentration near sight-line of Doppler beam}

$$
e=\left[\int \frac{F_{s}}{\gamma-\sqrt{\gamma^{2}-1} \cos (\theta)} d \theta\right]^{2}=\left[\frac{\left.\arctan \left(\sqrt{(} \gamma^{2}-1\right)+\gamma\right) * \tan (\theta / 2)}{\pi}\right]^{2}
$$

\section{REFERENCES}

[1] Schoedel, R. \& Genzel, R. \& Ott, T. \& Eckart, A.. (2003). The Galactic Center stellar cluster: The central arcsecond. Astronomische Nachrichten. 324. 10.1002/asna.200385048.

[2] K. Bell \& J. Schmelz, "Magnetic Field May Be Keeping Milky Way's Black Hole Quiet",NASA,www.nasa.gov/feature/1 field-may-be-keeping-milky-way-s-black-hole-quiet, accessed Mar 42020

[3] T. Opatrný, L. Richterekb, P. Bakala. "Life Under a Black Sun. American Journal of Physics 85, 14, , Appendix A8-A9 (2017) Available online at arxiv.org/abs/1601.02897

[4] J. B. Hartle, "Gravity: An Introduction to Einsteins General Relativity" pp 19(Addison Wesley, San Francisco, 2003).

[5] R. Lambourne, Relativity, Gravitation and Cosmology ch 5-6, Cambridge University Press (2010) Available online Relativity, Gravitation and Cosmology

[6] B. Ydri, Lectures on General Relativity, Cosmology and Quantum Black Holes, IOP Publishing Ltd (2017). Available online Lectures on ...

[9] V De Falco et al., "Approximate analytical calculations of photon geodesics in the Schwarzschild metric", Astronomy and Astrophysics, Vol. 595, Issue 6141 (2016). Available online AA 595

[10] G. Muñoz, "Orbits of massless particles in the Schwarzschild metric: Exact solutions", American Journal of Physics, Vol. 82, Issue 6, p.564-573 (2014). 
[11] F Rajabi et al., " A simple relationship for the spectro-temporal structure of bursts from FRB 121102", Monthly Notices of the Royal Astronomical Society 498, no. 4 (2020).

[12] A B Pearlman et al., "Unveiling the Spectro-temporal Behavior of Repeating Fast Radio Bursts at High Radio Frequencies", Bulletin of the American Astronomical Society, Vol. 53, No. 1 (2021).

[13] Bisnovatyi-Kogan et al., "Strong Gravitational Lensing by Schwarzschild Black Holes", Astrophysics, Vol. 51, No. 1 (2008). Available online Strong Gravitational Lensing by Schwarzschild Black Holes

\section{ACKNOWLEDGEMENTS}

I extend my gratitude to Pavel Bakala who encouraged me not to leave this paper in an unfinished and unpublished state, and who mentored my study of this subject and my development of these ideas. 